\title{
Direct generation of voltage and current by gas flow over Carbon nanotubes and semiconductors
}

\author{
A. K. Sood* and Shankar Ghosh \\ Department of Physics, Indian Institute of Science,Bangalore 560 012, India
}

\begin{abstract}
We report here a direct generation of measurable voltages and currents when a gas flows over a variety of solids even at the modest speed of a few meters per second. The underlying mechanism is an interesting interplay of Bernoulli's principle and the Seebeck effect: Pressure differences along streamlines give rise to temperature differences across the sample; these in turn produce the measured voltage. The electrical signal is quadratically dependent on the Mach number $M$ and proportional to the Seebeck coefficient of the solids. Results are presented for doped Si and Ge , single and multiwall carbon nanotubes and graphite. Our results show that gas flow sensors and energy conversion devices can be constructed based on direct generation of electrical signals.
\end{abstract}

Corresponding Author : * asood@physics.iisc.ernet.in

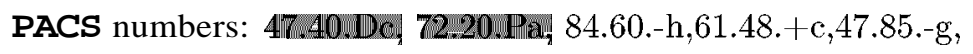


Recently it has been shown that the flow of liquids over single-wall carbon nanotubes $(\mathrm{SWNT})$ generates a voltage in the tubes along the flow direction $[1,2]$. The induced voltage has a logarithmic dependence on flow velocity over the entire range of velocities $10^{-7}$ to $10^{-1} \mathrm{~m} / \mathrm{s}$ covered in the experiments. This phenomenon is specific to the onedimensional nature of the nanotubes and is absent in graphite. A question naturally arises as to whether similar mechanisms can generate voltages in the carbon nanotubes due to the flow of gases as well? In this paper we show that an equally striking effect exists for gas flow as well but for a fundamentally different reason than the mechanisms operating for the liquid flow. Specifically, we show for the first time, the direct generation of voltage by the flow of common gases such as argon, nitrogen and oxygen over SWNT. Furthermore, the effect is not unique to nanotubes: A voltage is generated by the gas flow over a wide variety of solids, including single and multi-walled carbon nanotubes, doped semiconductors and metals and for a wide range of gas velocities [ the range covered in our experiments is 1 to $140 \mathrm{~m} / \mathrm{s}$. We also establish that the voltage and current depend quadratically on the flow velocity. The magnitude and sign of the voltage depends on the properties of the solid. For argon at a flow velocity of $11 \mathrm{~m} / \mathrm{s}$, the voltage generated is $-16.4 \mu \mathrm{V}$ for n-type Ge, whereas it is $5.9 \mu V$ for the sample of single walled carbon nanotubes (SWNT) bundles.

Figure 1(a) shows a schematic layout of the experimental set-up to achieve a calibrated gas flow velocity on the sample. The gas from a compressed gas cylinder (maximum pressure of $150 \mathrm{bars}$ ) is let out at a given pressure in a tube of diameter $7 \times 10^{-3} \mathrm{~m}$. The flow rate $\mathrm{Q}$ is measured using a rotameter as shown in Figure 1(a). The average velocity $u$ at the end of the tube with cross-sectional area $(\phi)$ is deduced from $\mathrm{Q}$ as $\mathrm{u}=Q / \phi$. The sample is kept at an angle $\alpha=\pi / 4$ with respect to the horizontal axis to achieve the optimal signal. As we show later, $\alpha=0$ " would produce no effect since the pressure gradient is zero, and $\alpha=\pi / 2$ too would give no signal due to symmetry. The results are similar when the sample is kept inside the tube (at a distance of $2 \times 10^{-2} \mathrm{~m}$ from the exit point) or $1 \times 10^{-2} \mathrm{~m}$ outside the tube.

We have used a variety of solids such as single crystals of n-type Ge (Sb doped, $\sigma=100$ / Rcrn), n-Si $(\sigma=100 /$ Rcrn), p-Si (a = 100/ $\mathrm{ccm})$, SWNT, multi-wall carbon nanotubes (MWNT), graphite and polycrystalline copper to establish the ubiquity of the gas flow induced voltage generation. The sizes of the semiconducting samples and copper are about $3 \times 10^{-3} \mathrm{~m}$ along the flow and $1 \times 10^{-3} \mathrm{~m}$ perpendicular to the flow. The electrical contacts for 
the semiconducting materials ( $\mathrm{Si}, \mathrm{Ge}$ ) was made with copper leads of $1.3 \times 10^{-4} \mathrm{~m}$ diameter using silver emulsion (shown by the shaded region of Fig. 1(b)). The exposed (active ) portion of the sample i.e not covered by silver emulsion is about $2 \times 10^{-3} \mathrm{~m}$ (marked as $d$ in Fig. 1(b)) along the flow and $1 \times 10^{-3} \mathrm{~m}$ perpendicular to the flow. The samples of SWNT, MWNT and graphite, prepared by densely packing the powder between two metal electrodes, were about $1 \times 10^{-3} \mathrm{~m}$ along the flow, $2 \times 10^{-3} \mathrm{~m}$ wide and $2 \times 10^{-4} \mathrm{~m}$ thick. Figure $1(\mathrm{c})$ shows the voltage across the $\mathrm{n}-\mathrm{Ge}$ sample as a function of time when the gas flow $(u=7 \mathrm{~m} / \mathrm{s})$ over the sample is switched on and off. Data for the steady flow voltage $V$ for a variety of samples and flow velocities is shown in Figs. 2 and 3. Proper electromagnetic interference shielded cables were used to measure the signals using a KEITHLEY 2000 multimeter.

It can be clearly seen from Figs 2 and 3 that for nitrogen gas over p-Si,n-Si, n-Ge, SWNT, MWNT and graphite, the voltage $\mathrm{V}$ generated varies as $u^{2}$ over a wide range of $u$ ( as does the current, not shown ). Figure 2 shows the fit to $V=D u^{2}$ with the fit parameter $D$ given in Table 1. This is even clearer in Fig. 3, which shows the same data plotted versus $M^{2}$, where $M$ is the Mach number given by $M=u / c, \mathrm{c}$ being the sound velocity( $=353 \mathrm{~m} / \mathrm{s}$ for nitrogen and $323 \mathrm{~m} / \mathrm{s}$ for argon at $300 \mathrm{~K}$ ). The use of $M^{2}$ is motivated by the theory to be presented later. The solid lines in Fig. 3 are fit to $\mathrm{V}=A M^{2}$, where $\mathrm{A}$ is a fitting parameter also given in Table 1. We have also done experiments using the polycrystalline copper sheet for which the slope $A$ is very small (see Table1). It can be seen that the sign of the signal for p-type $\mathrm{Si}$ and SWNT is opposite to that for n-type $\mathrm{Si}, \mathrm{n}-\mathrm{Ge}$, graphite and copper. SWNT samples are usually unintentionally p-doped, as reported in the literature [3,4], which can explain the sign of the flow induced voltage which was found to be same for SWNT and p-Si. The inset of Fig. 3 shows the plot of the slope A versus the known Seebeck coefficient $\mathrm{S}[3-8]$ of the samples of the same $d$, as given in Table 1 . The coefficient $A$ depends linearly on $\mathrm{S}$, as shown by the fitted line in the inset, with slope $=60 \mathrm{~K}$.

Figure 4 shows the generated voltage $\mathrm{V}$ over a large range of values of $M^{2}$ for the flow of argon (solid squares) and nitrogen gases (open circles) over $\mathrm{n}-\mathrm{Ge}$. A change in slope is clearly seen around $M^{2} \leq 0.05$. The inset in Fig.4 clearly shows that the slope A for $M^{2} \leq 0.05$ (called regime I) is higher for argon as compared to that for nitrogen. The ratio of slopes $A($ argon $) / A($ nitrogen $)=1.2$. That there are actually two $M^{2}$ - regimes is clear from our theoretical analysis of the mechanism behind the generation of electrical signal induced by 
the flow of gases over the solids and is discussed below.

For adiabatic steady inviscid flow of a gas, Bernoulli's equation gives the pressure difference along a streamline in terms of Mach number $\mathbf{M}$ as [9]

$$
\frac{P}{P_{0}}=\left[1-\frac{1}{2}(\gamma-1) M^{2}\right]^{\frac{\gamma}{\gamma-1}} \cong 1-\frac{\gamma}{2} M^{2} \quad\left(\text { for } \gamma M^{2} \ll 1\right),
$$

$\mathrm{y} \equiv C_{p} / C_{v}, ; C_{p}\left(C_{v}\right)$ being the specific heat at a constant pressure (volume). The values of $\gamma$ for argon and nitrogen are 1.667 and 1.404, respectively. In Eqn. $1 P_{0}$ is the maximum pressure at a point on the streamline where velocity is zero . Such a point is the leading edge on the surface of the sample past which the gas is moving and is called the stagnation point. For the sample geometry shown in Fig 1(b), the pressure difference between the two ends of the active sample exposed to the gas flow (i.e without the electrodes) is hence

$$
\frac{P_{L}-P_{R}}{P_{0}} \simeq \frac{\gamma}{2}\left(M_{R}^{2}-M_{L}^{2}\right)
$$

The subscripts $L(R)$ denote the left (right) of the active sample when the gas flows from left to right. From the ideal gas law, the fractional temperature difference $\Delta T / T$ is related to the pressure difference $\Delta P / P$ and the density difference $\Delta \rho / \rho$ as $\Delta T / T=\Delta P / P-\Delta \rho / \rho$. When $M<<1$, the change in density of the gas is negligible i.e. the fluid is essentially incompressible and hence $\Delta T / T=\Delta P / P$. Therefore, the temperature difference along a streamline between two points separated by a distance $d$ for $\mathrm{M} \ll 1$ (called regime $\mathrm{I}$ ) is

$$
\frac{\Delta T}{T_{0}}=\frac{\gamma}{2}\left(M_{R}^{2}-M_{L}^{2}\right)
$$

where $A T=T_{L}-T_{R}>0$. The gas flowing past the sample kept at an angle $\mathrm{Q}$ with respect to the horizontal axis corresponds to an accelerating flow and hence $M_{R}>M_{L}$. As shown by Falkner and Skan [10] the tangential component of the velocity $\mathrm{u}$ of the outer flow depends on the streamline distance $\mathbf{x}$ measured along the flat boundary as

$$
u(x) \propto u x^{\alpha /(\pi-\alpha)} .
$$

For our geometry $\alpha=\pi / 4$ and hence $u(x) \propto x^{1 / 3}$. The temperature difference along the streamline in the gas flow will induce a temperature difference in the solid along the flow direction. The temperature difference, in turn, will result in a voltage difference $\mathrm{V}$, defined as $V_{L}-V_{R}$, due to the Seebeck effect. We can write, 


$$
V=\frac{k}{2} T_{0} S \gamma\left(M_{R}^{2}-M_{L}^{2}\right)
$$

where $\mathrm{S}$ is the Seebeck coefficient of the solid, positive for p-type (majority carries as holes) and negative for n-type materials. The factor $k$ depends on the specific interactions between the gas and the solid surface as well as on the boundary conditions of the temperature difference between the gas and the solid. In non-rarefied gases, the boundary condition at the surface of a solid is that the temperatures of the gas and solid are equal[11], in which case $k=1$. But this boundary condition applies only if the mean free path of the gas molecules is vanishingly small[11, 12]. The factor $k$ takes into account all the differences from this ideal boundary condition. The present phenomenon of voltage generation by flow of gases is not applicable to the flow of the liquids where the viscous drag dominates.

Beyond a certain value of $M(\sim 0.2)$, called regime II, the density changes of the gas should be taken into account which gives [9]

$$
\frac{A T}{T_{0}} \simeq \frac{1}{2}(\mathrm{y}-1)\left(M_{R}^{2}-M_{L}^{2}\right)
$$

and hence

$$
V=\frac{k T_{0}}{2}(\gamma-1) S\left(M_{R}^{2}-M_{L}^{2}\right) \propto \frac{k T_{0}}{2}(\gamma-1) S M^{2}
$$

We now compare the predictions of our model with the experiments. (i) We note from Eqn. 4 that $M_{L}^{2} \propto M^{2} x_{1}^{2 / 3}$ and $M_{R}^{2} \propto M^{2}\left(x_{1}+d\right)^{2 / 3}$.Equations 5 and 7 , therefore, predict that in both regimes, the induced voltage should be proportional to $M^{2}: \mathrm{V}=A M^{2}$. This is in agreement with our experimental results shown in Figs. 2-4. (ii) The slope A should depend linearly on $\mathrm{S}: \mathrm{A}=k T_{0} S \gamma / 2$ in regime I (Eqn. 5). This is the case as shown by the plot of the observed values of A (solid circles) on the known values of $\mathrm{S}$ for different materials (Table 1)[13], in the inset of Fig.3. Using the fitted value of the slope $(=60 \mathrm{~K})$ together with $\gamma=1.404$ and $T_{0}=300 \mathrm{~K}, k$ turns out to be 0.28 . (iii) Equation 5 predicts that the ratio of the slopes, A, in the regime I for the flow of argon and nitrogen gases is $\gamma($ argon $) / \gamma($ nitrogen $)=1.2$, in exact agreement with the observed ratio (inset to Fig.4). Equations 5 and 7 suggest that the ratio of slopes in the regions I and II of Fig. 4 should be $\gamma /(\gamma-1)$. This compares well with the observed value of 3.44 for argon( filled squares). (iv) The inset of Fig. 2 shows the dependence of measured $V$ on the sample length 
$d$ for $\mathrm{n}-\mathrm{Ge}$ and flow velocity $\mathrm{u}=10 \mathrm{~m} / \mathrm{s}$. The data fit extremely well with the equation $V=a_{1}+a_{2}\left\{\left(1+d / x_{1}\right)^{2 / 3}-1\right\}$ (c.f Eqn.(4)),where $a_{1}$ and $a_{2}$ are fitting parameters [14] and $x_{1}=0.5 \mathrm{~mm}$ (from the sample geometry). The mechanism proposed by us justifies this dependence on $\mathrm{d}$ : from Eqn. 4 for $\mathrm{Q}=\pi / 4, u^{2} \propto d^{2 / 3}$. Therefore $V \propto\left[\left(x_{1}+d\right)^{2 / 3}-x_{1}^{2 / 3}\right]$. (v) For $\alpha=0^{\circ}$ and $\pi / 2$, we observe that the voltage is not generated by the gas flow and the signal changes sign for $\alpha>\pi / 2$ [15]. This observation is understandable from Eqn. 4 which predicts $u_{L}=u_{R}$ for $\alpha=0^{\circ}$, orthogonal stagnation points for $\alpha=\pi / 2$, and decelerating flow for $\mathrm{Q}>\pi / 2$. (vi) Another outcome of the proposed mechanism is that the voltage generated by the flow of gases on the platinum metal should be negligible because $\mathrm{S} \sim 0$ for platinum. This is indeed the case as shown by our experiments (see plus signs in Figs.2 and 3).

Our experiments clearly show that a sensor to measure the flow velocity of the gases can be made based on the generated electrical signal. It is an active sensor which gives direct electrical response to the gas flow. This should be compared with the widely used gas flow sensor based on thermal anemometry, wherein, the fluid velocity is sensed by measuring changes in heat transfer from a small, electrically-heated sensor (wire or thin film) exposed to the fluid. Thermal anemometry works on heat balance equations and hence any small changes in the temperature, pressure or composition of the gas can cause erroneous readings. Such effects are minimum or can be easily taken into account in the sensors based on the direct generation of flow-induced voltage or current in the sensor material.

Even though we have shown results only for a few solids we emphasize that the effect is not restricted to these materials alone. The guiding principle for the choice of the solid is its high Seebeck coefficient, which will suggest other solids like selenium $(\mathrm{S} \sim 900 \mu / K)$, tellurium ( $\mathrm{S} \sim 500 \mu \mathrm{V} / K$ ), GaAs, oxides and electrically conducting polymers. We have seen that for low doped semiconductors with $\sigma \sim 10^{-6} / \Omega \mathrm{cm}$, the voltage due to the flow is negligible, suggesting that the resistivity of the solid should not be very high. The magnitude of voltage and current can be easily scaled up by using a serial and parallel combination of sensing elements. Following Allison et.al [5, 6], another attractive idea is to take advantage of inverse Seebeck coefficients of $n$ and $\mathrm{p}$ type $\mathrm{Si}$ or Ge: the $\mathrm{n}$ and $\mathrm{p}$ type strips can be alternated and electrically bonded in series to add the individual Seebeck voltages when the sample is exposed to the gas flow. Our results also suggest that gas flow energy can be converted directly into the electrical signal without any moving part, thus having a potential 
TABLE I: Comparison of the slope $D$ and $\boldsymbol{A}$ for materials with different Seebeck Coefficients with respect to $\mathrm{Pt}$.

\begin{tabular}{|c|c|c|c|c|}
\hline Sample & $D\left(\mu V s^{2} / m^{2}\right)$ & $A(\mu V)$ & $\mathrm{S}(\mu \mathrm{V} / \mathrm{K})$ & Reference \\
\hline $\mathrm{n}-\mathrm{Si}$ & -0.28 & -35697 & -450 & {$[5]$} \\
\hline $\mathrm{p}-\mathrm{Si}$ & 0.14 & 14539 & 450 & {$[5]$} \\
\hline $\mathrm{n}-\mathrm{Ge}$ & -0.013 & -17576 & -548 & {$[5]$} \\
\hline $\mathrm{SWNT}$ & 0.04 & 5389 & 20 & {$[3]$} \\
\hline $\mathrm{MWNT}$ & 0.04 & 5538 & 20 & {$[7]$} \\
\hline graphite & -0.1134 & -1810 & -8 & {$[8]$} \\
\hline copper & 0.0002 & 23 & 7.4 & {$[5,6]$} \\
\hline
\end{tabular}

for applications in generating electricity[16].

We thank Profs. Rama Govindrajan, S. Ramaswsmy and N. Kumar for useful discussions. Thanks are due to Prof. C. N. R. Rao and Dr. A. Govindaraj for nanotube samples and Dr. V. Venkataraman for silicon samples. AKS thanks the Department of Science and Technology ( Government. of India) for financial support.

[1] S. Ghosh, A. K. Sood and N. Kumar, Science 299, 1042 (2003).

[2] P. Kral and M. Shapiro, Phys. Rev. Lett. 86, 131 (2001).

[3] P.G. Collins, K. Bradley, M. Ishigami, A. Zettl, Science $287,1801(2000)$.

[4] J. Hone, I. Ellwood, M. Muno, A. Mizel, M.L. Cohen, A.Zettl, A.G. Rinzler and R.E.Smalley, Phys. Rev. Lett. 80, 1042 (1998).

[5] S.C. Allison, R.L. smith, D.W. Howard, C. González, S.D.Collins, Sensors and Actuators A, 104,32 (2003).

[6] D. M. Rowe, CRC Handbook of thermoelectrics, Boca Raton, FL: CRC Press, 1995.

[7] R.Seshadri, H.N.Aiyer, A.Govindaraj and C.N.R.Rao, Solid State Commun. 91, 195(1994).

[8] T.Takezawaetal, Phil. Mag. 19,623(1969).

[9] L.D. Landau and E. M. Lifshitz, Fluid Mechanics, 2nd Edition , Butterworth Heinemann, 
p.319 (1998).

[10] V.Falkner and S.Skan ,Phil. Mag., 12,865(1931);

C. Pozrikidis, Introduction to Theoretical and computational Fluid Dynamics, Oxford University Press, p.401 (1997).

[11] L.D. Landau and E. M. Lifshitz, Physical Kinetics, 2nd Edition, Butterworth Heinemann , p.51 (1998).

[12] At very high velocities, the gases become rarefied, i.e., the mean free path $(l)$ of the gas molecules has to be taken into account which results in correction, first order in "l/Charecteristic length", to the temperature between the gas and the solid. This will influence the parameter $k$ in Eqn.5. This has now been added as ref (12) in the revised manuscript. Eqn.(5).

[13] The values of the Seebeck coefficient used for Fig.3(inset) are some typical values of n-type Si $(p=0.0035 \Omega-c m)$, p-type Si $(p=0.0035 \Omega-c m)$ and n-type $\operatorname{Ge}(\rho=0.069 \Omega-e r n)$. The exact values of the Seebeck coefficient for the materials used in our experiments are expected to be slightly different.

[14] $a_{1}=17 \mu V$ and $a_{2}=2.5 \mu V$. The experiments on length dependencies were done on a single crystal of Germanium by exposing different lengths (i.e., varying distances between the electrodes) of the crystal surface to the gas flow. The non-zero value of $a_{1}$, ie., $\mathrm{V} \neq 0$ for $d=0$ arises from poor thermal insulation of the unexposed part of the crystal to the gas flow.

[15] The variation of $V$ as a function of $\alpha$ for a fixed value of $u=11 \mathrm{~m} / \mathrm{s}$ was measured for a few values of $\mathrm{Q}: \mathrm{Q}=25^{\circ}, \mathrm{V}=10.8 \mu \mathrm{V}, \mathrm{Q}=35^{\circ}, \mathrm{V}=17.4 \mu \mathrm{V}$ and for $\mathrm{Q}=45^{\circ}, \mathrm{V}=18.2 \mu \mathrm{V}$.

[16] The open-circuit voltage is related to the short-circuit current by the sample resistance. However,the detailed issues of efficiency is to be addressed for this effect. 


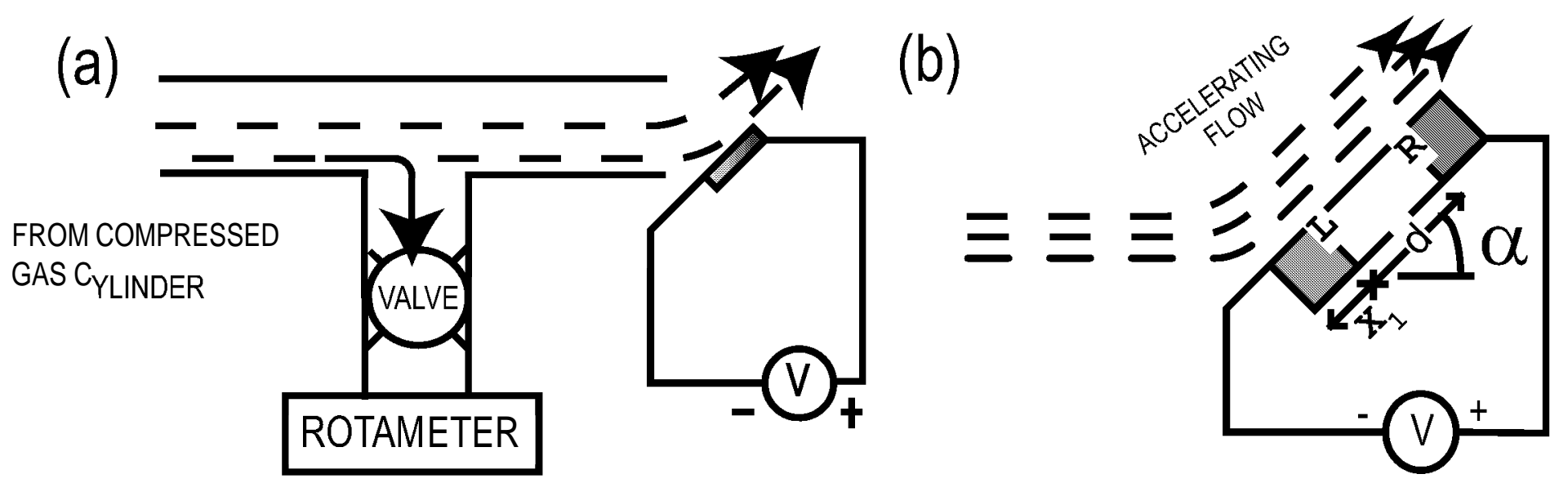

(c)

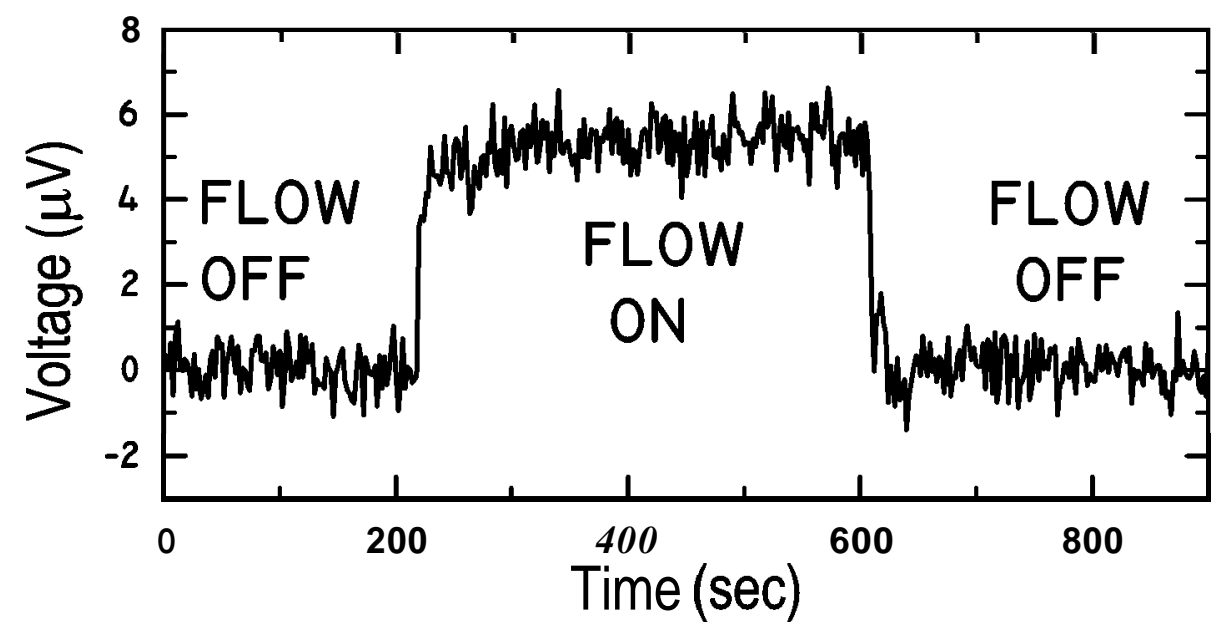

FIG. 1: (a) Schematic of the experimental setup. The flow rate at the exit point is deduced from the measured flow rate at the side port using the rotameter. (b) Sample: shaded portions mark the electrodes. The positive terminal of the voltmeter is connected to the right $(\mathrm{R})$ of the sample of active length $\mathrm{d}$ kept at an angle $\alpha=\pi / 4$ with respect to the horizontal axis.(c) Typical response of $\mathrm{n}$ doped $\mathrm{Ge}$ for a flow of argon gas at $7 \mathrm{~m} / \mathrm{sec}$. 


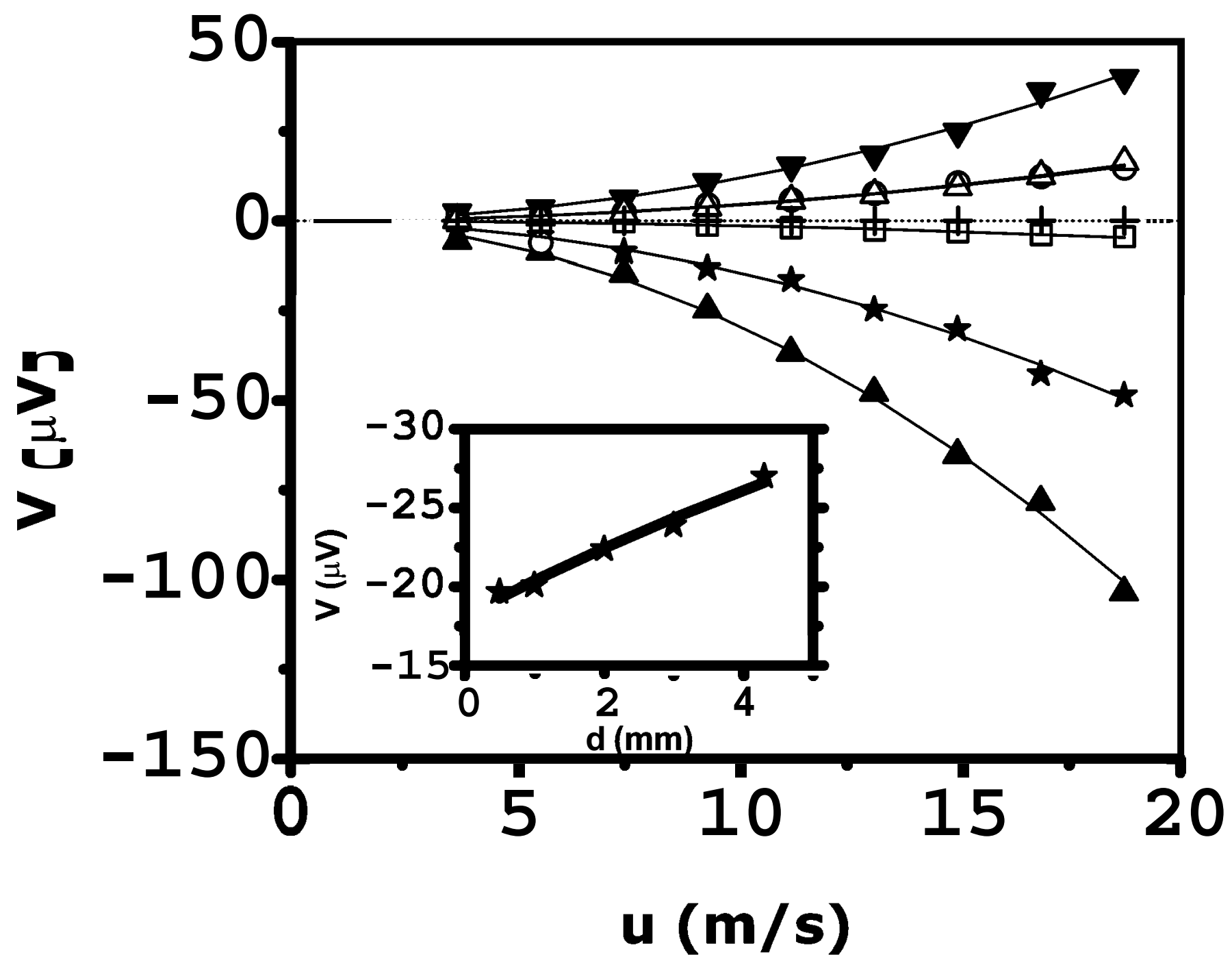

FIG. 2: Dependence of the signal V on the flow velocity of nitrogen for (bottom to top) $\mathrm{n}-\mathrm{Si}$ ( filled up triangles), $\mathrm{n}-\mathrm{Ge}$ ( stars), graphite (open squares), Pt metal (plus), SWNT(open circles) , MWNT ( open up triangles) and p-Si ( filled down triangles). The solid lines are fit to $\mathrm{V}=D u^{2}$, where $D$ is the fitting parameter given in Table 1 . Inset shows the dependence of $\mathrm{V}$ for $\mathrm{n}-\mathrm{Ge}$ as a function of the active length $d$ for a fixed value of $u$. The solid line is a fit to the equation $\mathrm{V}=a_{1}+a_{2}\left\{\left(1+d / x_{1}\right)^{2 / 3}-1\right\}$ where $a_{1}$ and $a_{2}$ are fitting parameters [14]. 


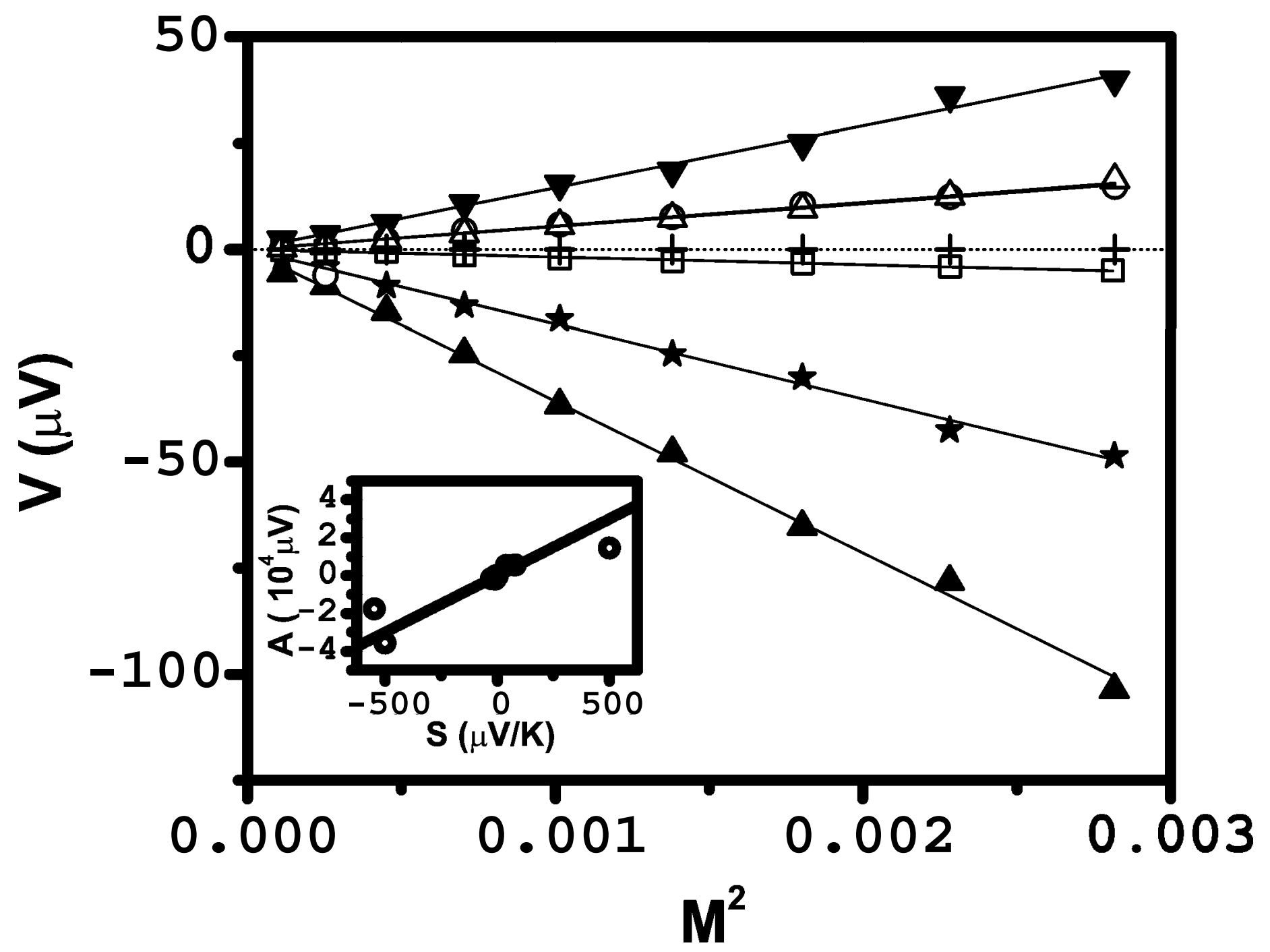

FIG. 3: Plot of $\mathrm{V}$ versus square of the Mach number $M$ for the flow of nitrogen gas . Data and symbols are same as in Fig.2 . Solid lines are a fit to $\mathrm{V}=A M^{2}$, where $A$ is a fitting parameter given in Table 1 . The inset shows the dependence of $\boldsymbol{A}$ on the the seebeck coefficient $S$ and the line is a linear fit with the slope of $60 \mathrm{~K}$. 


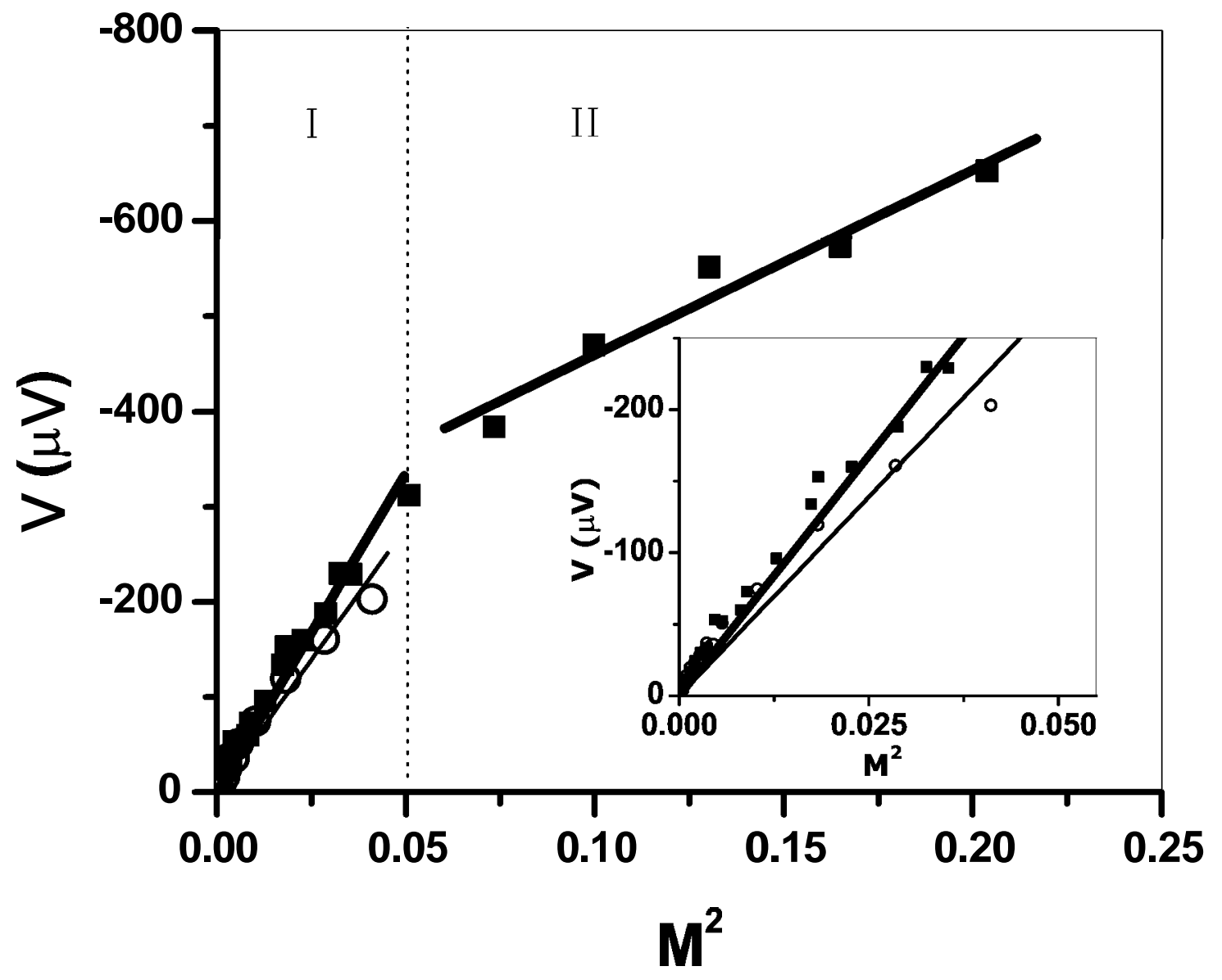

FIG. 4: V versus $M^{2}$ for the flow of argon( filled squares ) and nitrogen (open circles). The lines show fit to linear equation. The inset shows the expanded plot of regime I. 